\title{
REVIEWERS
}

\section{CONFERENCE REVIEWERS}

\begin{tabular}{ll}
\hline Neal Ashkanasy & Miriam Matteson \\
Yevgen Bogodistov & Minoo Miri \\
Prisca Brosi & Christiana Mitcheva \\
Arik Cheshin & Jennifer Nash \\
Catherine Daus & Shazia Nauman \\
Rebecca Dickason & Usha Pappu \\
Alana Dorris & Frances Peart \\
Tonya Ensign & Catherine Prentice \\
Riikka Harikkala-Laihinen & Tamilselvan Ramis \\
Silja Hartmann & Vishal Rana \\
Paul Harvey & Phatcharasiri Ratcharak \\
Rosalie Holian & Faryal Razzaq \\
Ronald Humphrey & Adam Robertson \\
Gemma Irving & Stuti Saxena \\
Moyi Jia & Samantha Sim \\
Bob Jones & Jeffrey Spence \\
Peter Jordan & Eugene Yu Jin Tee \\
Virginie Kidwell & Audrey Yea Ling Teh \\
Hector Madrid & Dorit Treister \\
David Martinez-Iñigo & Ashlea Troth \\
\hline
\end{tabular}

\section{ADDITIONAL REVIEWERS ${ }^{1}$}

Jasmin C. R. Härtel

${ }^{1}$ Reviewers who completed "friendly reviews" of revised chapters 